Sociologie et sociétés

\title{
La socialisation économique en Suisse
}

Récits rétrospectifs sur le rôle des parents et des enfants

durant l'enfance et l'adolescence

Economic Socialization in Switzerland

Retrospective Accounts of a Shared Experience. The role of
Parents and Children during Childhood and Adolescence

\section{Caroline Henchoz, Francesca Poglia Mileti et Fabrice Plomb}

Volume 46, numéro 2, automne 2014

URI : https://id.erudit.org/iderudit/1027151ar

DOI : https://doi.org/10.7202/1027151ar

Aller au sommaire du numéro

\section{Éditeur(s)}

Les Presses de l’Université de Montréal

ISSN

0038-030X (imprimé)

1492-1375 (numérique)

Découvrir la revue

Citer cet article

Henchoz, C., Poglia Mileti, F. \& Plomb, F. (2014). La socialisation économique en Suisse : récits rétrospectifs sur le rôle des parents et des enfants durant

l'enfance et l'adolescence. Sociologie et sociétés, 46(2), 279-299.

https://doi.org/10.7202/1027151ar

\section{Résumé de l'article}

La socialisation économique est un sujet peu étudié par la sociologie économique, celle de la jeunesse et de la socialisation. Nous l'abordons ici à partir d'un point de vue original, celui des « socialisés » eux-mêmes, ce qui permet de mettre en évidence la part active qu'ils prennent à ce processus. L'analyse de plus de 40 entretiens portant sur l'enfance et l'adolescence d'hommes et de femmes de Suisse francophone aujourd'hui âgés entre 25 et 40 ans met en lumière trois composantes essentielles de l'apprentissage du rapport à l'argent : l'éducation parentale, l'observation et l'expérimentation. Nous montrons que les jeunes apprennent très tôt à devenir des consommateurs et des gestionnaires prudents mais aussi des producteurs de revenus. L'accent porté sur des valeurs comme l'épargne et le travail contribue peut-être à expliquer pourquoi la Suisse est un pays dont le taux d'endettement des ménages est parmi les plus faibles d'Europe. 


\section{La socialisation économique en Suisse}

Récits rétrospectifs sur le rôle des parents et des enfants durant l'enfance et l'adolescence

\section{CAROLINE HENCHOZ}

Université de Fribourg

Département des sciences sociales

Bd de Pérolles 90

Bureau $\mathrm{G}_{328}$

1700 Fribourg

Suisse

Courriel: caroline.henchoz@unifr.ch

\section{FRANCESCA POGLIA MILETI}

Université de Fribourg

Département des sciences sociales

Bd de Pérolles 90

Bureau $\mathrm{G}_{329}$

1700 Fribourg

Suisse

Courriel: francesca.poglia@unifr.ch

\section{FABRICE PLOMB}

Université de Fribourg

Département des sciences sociales

Bd de Pérolles 90

Bureau $\mathrm{G}_{333}$

1700 Fribourg

Suisse

Courriel: fabrice.plomb@unifr.ch

'objectif De Cet ARTicle est de répondre à deux questions que la sociologie éco-
nomique et la sociologie de la jeunesse et de la socialisation n'abordent que partiellement: qu'est-ce que les parents apprennent à leurs enfants en matière de pratiques économiques et comment est-ce qu'ils le leur enseignent? Répondre à ces questions en nous appuyant sur la perception et les souvenirs des enfants, aujourd'hui devenus adultes, met en lumière l'active participation des socialisés à leur propre socialisation. En effet, le processus d'apprentissage échappe en partie aux parents. Les enfants ne se contentent pas de ce qu'on leur inculque, ils piochent aussi un certain nombre d'informations dans leur entourage. En outre, les parents favorisent davantage l'apprentissage 
par l'expérimentation que par la transmission orale. En ce sens, le processus de socialisation économique est une expérience partagée qui implique la participation de différents acteurs. Dans cet article, nous présenterons plus particulièrement le rôle et la contribution de deux d'entre eux: les parents et leurs enfants.

En Suisse comme ailleurs, le processus de socialisation économique s'est fortement modifié au cours du xx ${ }^{\mathrm{e}}$ siècle. Jusqu'aux années 1960 pour les classes les plus modestes, l'enfant apprenait très jeune à devenir un pourvoyeur de revenus (Praz, 2005; Zelizer, 1994). C'était un membre «économiquement utile» de la famille qui permettait aux parents, et surtout aux mères chargées de récolter les paies, d'équilibrer le budget courant (Henchoz et Poglia Mileti, 2012). En ce sens, l'accès à un revenu ne conduisait pas pour autant les enfants à bénéficier d'indépendance économique. La remise de paie aux parents impliquait que ces derniers conservaient tout le pouvoir de décision.

La législation sur le travail des enfants et l'école obligatoire introduite dans tous les cantons suisses au début du $\mathrm{xx}^{\mathrm{e}}$ siècle, ainsi que l'allongement des études durant la première moitié $\mathrm{du} \mathrm{xx}^{\mathrm{e}}$ siècle ${ }^{1}$, ont contribué à limiter leur rôle en tant que producteurs de revenu. À partir du $\mathrm{xx}^{\mathrm{e}}$ siècle, et de sa dernière moitié pour les classes modestes, ce ne sont plus les enfants qui versent de l'argent à leurs parents mais, à l'inverse, les parents qui leur en donnent notamment sous forme d'argent de poche.

Ce passage de «l'enfant utile à l'enfant précieux» ou coûteux (Praz, 2005; voir aussi Zelizer, 1994) a fortement marqué la littérature contemporaine. Le rapport que les jeunes entretiennent à l'argent y est en effet essentiellement abordé par le prisme de l'argent de poche et de la consommation. Ainsi, la popularité des études de marché et plus récemment les inquiétudes concernant l'endettement des jeunes ont débouché sur une abondante documentation autour de l'enfant consommateur et sur une relative absence d'études sur leurs revenus et sur leur capacité à les gérer (Berti et Bombi, 1988).

\section{LE JEUNE CONSOMMATEUR, SES PARENTS ET SON ARGENT}

Pour les raisons déjà évoquées, les études sur la socialisation économique se sont surtout penchées sur la socialisation du jeune consommateur (Beutler et Dickson, 2008; Ward, 1974). Ainsi, un certain nombre de recherches mettent en évidence le rôle central de la consommation dans la formation de l'identité juvénile (Deutsch et Theodorou, 2010). La consommation serait perçue par les jeunes comme un moyen d'affirmer leur identité et leur appartenance à un groupe de pairs partageant un même style de vie. En Suisse comme ailleurs, le shopping serait devenu le loisir principal des jeunes, et plus particulièrement des jeunes filles (Bamert et Oggenfuss, 2005; Walser et Killias, 2009). Ce mode de vie porté sur la consommation engendrerait des dérives comme la consommation compulsive et son corollaire, l'endettement (Streuli et al., 2008). En Suisse, ce sombre constat est toutefois nuancé par des études récentes indi-

1. Dictionnaire historique de la Suisse, www.hls-dhs-dss.ch/index.php? lg=f, page consultée le 3 mars 2012. 
quant que les jeunes ménages de moins de 25 ans ne sont statistiquement pas plus endettés que leurs aînés de moins de 50 ans (Fleury et Christin, 2011; Henchoz et Wernli, 2012).

Le rôle socialisateur des parents a été étudié essentiellement par le biais de l'argent de poche. La socialisation économique y est généralement abordée dans une perspective durkheimienne (Durkheim, 1968 [1922]), soit comme un processus éducatif mené par les parents et visant, par le versement d'argent, à initier le futur consommateur à l'autonomie ${ }^{2}$ et à la responsabilité individuelle. Selon Furnham (1999), on retrouve les mêmes tendances dans différents pays. Introduits très tôt, les montants versés par les parents augmentent avec l'âge des enfants et leur niveau scolaire. On observe ainsi un transfert progressif des responsabilités et du pouvoir de décision (Barnet-Verzat et Wolff, 2001: 60). Les enfants prennent ainsi en charge de plus en plus de dépenses assumées autrefois par les parents (par exemple, l'habillement ou les déplacements).

L'influence de facteurs comme la classe sociale des parents ou le sexe de l'enfant sur le versement d'argent de poche reste sujet à controverse. Pour les uns, les règles concernant l'argent de poche diffèrent peu selon les classes sociales et les cultures (cf. les travaux comparatifs de Barnet-Verzat et Wolff, 2001; Furnham, 2001). Pour les autres, plus le revenu des parents est élevé, plus les enfants reçoivent des montants élevés (Streuli et al., 2008 pour la Suisse). Enfin, selon certains, les enfants des classes populaires recevraient plus d'argent de poche mais celui-ci couvrirait plus de dépenses, ce qui n’impliquerait dès lors pas plus d'indépendance (O'Brien, 1995). Concernant des différences de versement d'argent de poche entre les filles et les garçons, les constats sont également nuancés. Pour les uns (Furnham, 2001), le principe d'égalité prime. Pour les autres, les filles sont plus nombreuses que les garçons à recevoir de l'argent de poche jusqu'à 18 ans (Barnet-Verzat et Wolff, 2001: 58). La question de savoir si l'argent de poche fait partie d'un échange entre les parents et les enfants reste également débattue. Pour certains, l'argent de poche est souvent utilisé pour encourager le travail ménager et scolaire des jeunes (Barnet-Verzat et Wolff, 2001; 2008). O’Brien (1995) ne note toutefois pas d'échange entre gratitude, amour, attention et versement d'argent de poche: ce n'est ni attendu par les parents ni ce que les enfants ressentent.

L'apport de ces recherches reste limité. La plupart des études sur la socialisation économique en offrent une perspective plus psychologique que sociologique (Beutler et Dickson, 2008). Elles se concentrent essentiellement sur des actes de consommation et elles retiennent surtout le point de vue des parents, rarement celui des enfants. Enfin, la socialisation économique est essentiellement analysée sous l'angle éducatif. L’originalité de cet article est, d'une part, d'aborder l'apprentissage de l'activité économique en distinguant trois aspects : la production de revenus, leur gestion et leur utilisation; d'autre part, de considérer le processus de socialisation économique à partir

2. L'autonomie est définie ici comme la capacité qu'a un individu de se donner ses propres lois (par exemple, dans la gestion des relations amicales ou de son emploi du temps). L'indépendance fait référence à l'indépendance économique, soit au fait de disposer de ressources financières suffisantes pour ne pas dépendre d'autres personnes (Galland, 2009; de Singly, 2000). 
du point de vue des socialisés eux-mêmes, ce qui permet d'aborder la question de la socialisation autrement qu'en termes d'éducation parentale et de rendre compte du rôle des enfants dans ce processus.

\section{CONCEPTUALISATION DE LA SOCIALISATION ÉCONOMIQUE}

La socialisation économique se réfère de manière générale au processus par lequel l'enfant assimile des connaissances et des raisonnements concernant le monde économique (Roland-Levy, 2004). Comme l'ont déjà souligné Berti et Bombi (1988), l'apprentissage de l'acquisition de ressources économiques et de leur gestion a été peu exploré par la littérature scientifique. Celle-ci porte essentiellement sur la manière dont les ressources économiques sont dépensées. Or, les travaux de Zelizer (2005), et notamment son concept de «marquage de l'argent», remettent en question la distinction entre production, gestion et consommation des ressources économiques. Les individus différencient l'usage de l'argent selon sa provenance. Par exemple, l'argent reçu en héritage ne sera pas dépensé de la même manière que l'argent provenant d'un emploi. À l'image de jeunes qui cherchent un petit emploi pour financer leur désir d'achats, on observe également que la consommation a un impact sur la production de revenus.

Se fondant sur les théories de Piaget (1967), les études sur la socialisation économique offrent toutefois plusieurs pistes théoriques intéressantes, notamment sur le caractère processuel et interactif de la socialisation économique. Différents stades dans le développement et l'acquisition de connaissances économiques sont relevés (RolandLevy, 2004). Avec l'âge et l'expérience de l'enfant, on observe un progrès graduel dans l'étendue de ses connaissances et dans la manière dont il les organise (Leiser, 1984; Sonuga-Barke et Webley, 1993). Les enfants seraient d'abord des consommateurs et des prescripteurs d'achat au sein de la famille. Progressivement, ils développeraient leur sens critique et acquerraient de nouveaux savoirs de plus en plus abstraits concernant la consommation et l'économie (Bullock et al., 1993; Lea et al., 1995). Ainsi, plutôt que de distinguer socialisation primaire et secondaire, ce qui introduit une discontinuité et une différenciation entre des périodes où l'individu serait re/modelé par l'action de la société et d'autres où il agirait et où se manifesterait l'actualisation des produits de socialisation (Darmon, 2006: 98), nous reprendrons la notion de socialisation continue. «Étant donné la diversité des instances et mécanismes socialisateurs à l’œuvre, la variation des calendriers socialisateurs, elle-même produite socialement, et enfin la force indéniable de la société dans son modelage des individus, il semble beaucoup plus réaliste de présupposer une continuité de l'action de cette dernière» (Darmon, 2006: 98). Cette notion permet d'intégrer dans notre réflexion un autre apport central de Piaget: l'interactivité. Dubar (1991) considère ainsi la socialisation comme une suite d'interactions auxquelles les individus participent en interprétant, adhérant ou rejetant en fonction de leur expérience les normes et les valeurs qu'on leur transmet. Cela nous conduira à considérer plusieurs modalités de la socialisation: 
- La «socialisation-inculcation» (Lahire, 2002) concerne le processus de diffusion et d'inculcation de normes culturelles, de valeurs ou de modèles. Elle prend la forme d'injonctions, notamment de la part des parents, concernant les manières de voir et de dire le monde (Darmon, 2006: 109).

- La socialisation par expérimentation, par «entraînement ou pratique directe» (Lahire, 2002), se réfère à des faits spécifiques expérimentés individuellement, comme la consommation ou la recherche de revenus.

- La socialisation par l'observation et l'écoute souligne également le rôle actif des socialisés. Hochschild (2003) parle ainsi des oreilles indiscrètes des enfants (children as eavesdroppers) comme faisant partie intégrante du processus de socialisation. Par l'observation et l'écoute des interactions de leur entourage, les enfants « entrevoient le monde» (Goffman, 1996). Ils se font une image de leur environnement, de son fonctionnement et de la place qu'ils y occupent. La diversité des sources d'informations et la variété de savoirs impliqués expliqueraient pourquoi le produit de la socialisation est différent selon les individus, fragmentaire, voire inconsistant (Leiser, 1984).

\section{MÉTHODOLOGIE}

Quarante-quatre entretiens individuels biographiques de type compréhensif ont été effectués entre 2005 et 2009 auprès des membres de vingt-deux couples de Suisse francophone âgés de 25-45 ans, mariés et avec des enfants à charge ${ }^{3}$. Il s'agit de couples de classe moyenne dans le sens où les revenus cumulés des hommes et des femmes correspondent au revenu moyen en Suisse. Dans la plupart des couples, les femmes occupent un emploi à temps partiel et les hommes à plein temps, ce qui est, en Suisse, le mode de répartition du travail le plus fréquent dans les familles avec enfant ${ }^{4}$.

Les entretiens individuels visaient à comprendre la manière dont se construisent les usages et significations de l'argent durant le parcours de vie, de l'enfance à l'âge adulte. Dans cet article, nous nous penchons plus particulièrement sur l'enfance et l'adolescence des personnes interrogées et sur leur rapport à l'argent durant cette période. Ce découpage du cycle de vie n'a pas été défini par des bornes d’âge spécifiques. Les questions relativement ouvertes (comment vos parents géraient l'argent, avez-vous des anecdotes sur le sujet, quand avez-vous gagné votre premier revenu, etc.) laissaient aux témoins la possibilité de nous faire part des événements marquants de cette période. Dans la plupart des récits, le rôle central et différencié des pères et des mères dans la socialisation économique est apparu de manière évidente. Les expériences que nous relatons ici portent jusqu'à l'âge de 17-18 ans environ. L'influence des

3. Entretiens individuels effectués dans le cadre de la thèse de doctorat de C. Henchoz (2008a) et dans le cadre d'une recherche FNS, «Les significations et usages sociaux de l'argent au sein de trois générations» (requérante principale: F. Poglia Mileti; responsable de la recherche: C. Henchoz). Des entretiens de couple, non traités ici, ont complété ces entretiens individuels.

4. www.bfs.admin.ch/bfs/portal/fr/index/themen/20/05/blank/key/erwerbstaetigkeit/teilzeitarbeit. html, page consultée le 9 février 2012. 
parents au niveau économique ne s'arrête bien sûr pas à cet âge. Pour ceux qui ont poursuivi leurs études, elle est encore bien présente par la suite. En outre, les parents soutiennent régulièrement leurs enfants, notamment par des cadeaux ou des dons, à l'arrivée de leurs petits-enfants ou encore à l'achat d'un appartement ou d'une maison (Henchoz, 2008a). Nous avons toutefois choisi de nous arrêter autour de cette limite d'âge, car ensuite, l'intervention des parents vise moins la transmission d'un savoir, de valeurs ou de compétences économiques qu'un soutien financier. Dix-sept ou dix-huit ans est aussi l'âge où un certain nombre de personnes interrogées entrent en apprentissage, bénéficiant ainsi d'un revenu régulier, ce qui les amène à devenir plus indépendantes financièrement (Plomb, 2005).

Ce récit rétrospectif sur sa propre vie comporte un certain nombre de limites. Ainsi, il ne peut être assimilé à l'ensemble des expériences vécues par l'individu. Il n'en est que le reflet filtré par la mémoire, l'interprétation, la sélection et la hiérarchisation (Lemieux et Mercier, 1989). En entrevue, il renvoie aux questionnements de l'enquêteur ou à une grille thématique qui l'oriente (Lemieux et Mercier, 1989: 39). De même, c'est une forme de présentation de soi au sens de Goffman qui fait écho à des valeurs particulières, un désir de présenter une unité de vie, une congruence ou une adéquation avec les normes actuelles. En effet, ces récits font référence à un contexte historique spécifique qu'il conviendra de rappeler, l'enfance et l'adolescence des personnes interrogées s'étant déroulées pour la majorité d'entre elles durant les années 19701980. Enfin, nos données ne permettent pas de saisir l'impact de la classe sociale sur le processus de socialisation économique. D'une part, les personnes interrogées connaissent rarement le revenu parental et d'autre part, lorsqu'elles l'évaluent, elles le catégorisent comme un revenu moyen. En ce sens, ce que nous décrivons ici semble surtout se référer aux pratiques de la classe moyenne.

Le recueil de récits de vie a toutefois des avantages multiples (Bertaux, 1997; Lemieux et Mercier, 1989). Il permet de récolter des observations assorties de réflexions personnelles. Ainsi, revenir sur son enfance offre l'opportunité aux personnes interrogées de faire preuve de réflexivité. En se déployant dans le temps, le récit de vie rend compte de la dynamique propre à la socialisation et aux pratiques très concrètes qui en constituent le socle. Comme l'a montré Lahire (2002), les sollicitations produites par l'entretien permettent d'accéder à la variété des dispositions conscientes et inconscientes acquises durant l'enfance et l'adolescence. La comparaison des récits met en évidence des pratiques récurrentes offrant ainsi la possibilité d'opérer une certaine généralisation et ainsi de proposer un portrait dynamique du rôle des pères, des mères et des enfants dans le processus de socialisation économique qui a lieu durant cette période spécifique du cycle de vie.

La documentation citée dans cet article a été utilisée pour éclairer par la comparaison les phénomènes repérés dans les entretiens. L'interaction constante entre théories et données empiriques a été mobilisée durant toutes les étapes de la recherche, de la formulation de la question de recherche aux analyses et interprétations des données (Glaser et Strauss, 1967). Sans prétendre à la représentativité, cette approche favorise 
une perspective et un point de vue originaux sur le rôle des parents et des enfants dans la socialisation économique. L'absence de documentation sur le sujet nous a conduits à privilégier les extraits d'entretien dans la présentation qui suit. Ils permettent en effet d'éclairer le processus de socialisation et la spécificité du rapport que les Suisses francophones de classe moyenne interrogés ici entretiennent à l'argent.

\section{LES MÉCANISMES DE LA SOCIALISATION ÉCONOMIQUE DANS LA SPHÈRE FAMILIALE}

\section{Peu «d'éducation »}

L'argent n'est pas un sujet public en Suisse. Dell'Orto et Doyle (2001) recensent ainsi peu de documentation traditionnelle sur l'argent et la propriété. La tradition suisse la plus connue en matière d'argent est celle des banques et du secret bancaire. Les salaires et le niveau de vie sont des informations qui relèvent du domaine privé, comme le relève Dunand (1998: 165) dans son ouvrage Dessine-moi un Suisse:

Le Suisse $e^{5}$ évoque volontiers le prix des choses, du pain, du vin, des terrains, il échange avec discrétion — voitures, vêtements, vacances — les signaux codés de son niveau de vie, mais il évite soigneusement de parler de richesse et surtout de la sienne. Le salaire du voisin se mesure par comparaison, s'évalue à quelques détails ou se déduit du comportement de sa femme; jamais on ne lui pose la question: combien gagnez-vous?

Si l'argent est une affaire privée, ce n'est toutefois pas un sujet qu'on aborde pour autant au sein de la famille. Les personnes interrogées relèvent peu de transmission explicite concernant l'argent et son usage. La plupart d'entre elles ignorent le salaire de leurs parents ou encore comment l'argent était géré au sein de la famille. On est loin de la socialisation-éducation au sens de Durkheim relevée par les recherches sur l'argent de poche.

Nicolas: Je n'ai jamais su combien mon père gagnait. Mon père était assez ouvert là-dessus. Ma mère pas trop [...]. Mon père, il nous l'aurait dit volontiers. Ma mère, elle ne voulait pas. Mais il n'avait pas des revenus fixes comme médecin non plus.

Question: Vous lui avez posé la question?

Nicolas: Ouais, ouais mais mon père, il ne parle pas beaucoup. C'était toujours ma mère qui répondait pour lui: «Ouh! On ne gagne pas beaucoup!»

L'argent est une affaire privée qui, au sein de la famille nucléaire, relève de la sphère conjugale, voire de la sphère personnelle, car ce n'est pas forcément un sujet qui suscite beaucoup de discussions au sein des couples (Henchoz, 2008a).

5. Les auteurs cités font référence aux «Suisses» sans préciser leur appartenance sociale, de genre ou de régions linguistiques. Ces propos nous semblent toutefois révélateurs du rapport à l'argent qu'entretiennent les Suisses romands interrogés, c'est pourquoi nous avons choisi de les faire figurer dans cet article. 
On ne parlait pas beaucoup d'argent dans la famille. Ce n'est pas un truc, ce n'était pas tabou mais ce n'était pas... C'était quelque chose [...] pour les grands. Puis toi, tu es un petit peu petit. (Annie)

Cette ségrégation entre le monde des adultes et celui des enfants se confirme par le fait que les discussions relevées autour de l'argent ont lieu bien plus tard dans le parcours de vie, une fois que les enfants sont majeurs. Ce n'est qu'après les histoires d'endettement du frère que les parents d'Agnès regrettent de leur avoir «transmis trop tardivement la valeur de l'argent ». Ce n'est qu'après le décès de son mari que la mère d'Alice lui apprend que celui-ci était dépensier et que la situation était difficile.

La transmission semble peu passer par les mots. «Je ne dirais pas que c'est une transmission: "Mon fils quand tu seras grand, tu devras faire comme ça » ", relève Tom. «Les choses se sont faites un peu naturellement», renchérit Danièle. Malgré cette absence d'explicitation des pratiques économiques, l'écrasante majorité des personnes interrogées relèvent avoir reçu le même message de leurs parents:

Nous avons appris que l'argent se gagnait, qu'il ne faut pas avoir de dettes, que c'est bien plus facile d'en dépenser que d'en gagner. Dans le sens que c'est dur de gagner de l'argent et qu'il y a beaucoup de tentations pour le dépenser facilement. (...) Très vite on a appris ce que c'était, la valeur de l'argent. (Cathy)

Si elle ne passe pas par la parole, comment la «valeur de l'argent», pour reprendre les termes de Cathy, est-elle transmise par les parents? Nous relevons ici deux mécanismes centraux de la socialisation: l'observation et l'expérimentation.

\section{L'observation}

Si peu de personnes interrogées mentionnent une transmission orale, nombreuses sont celles qui insistent sur leur ressenti:

Mes parents n'en parlaient pas. Je n'ai pas ressenti [les auteurs soulignent] de difficultés. Ce n'était pas un sujet. Devant moi, ce n'était pas abordé. (Sandra)

Comme l'illustrent les propos ci-dessous, le ressenti se construit à partir de deux processus complémentaires: l'observation et la comparaison.

Question: [Votre mère] vous avait fait part des difficultés qu'elle avait?

Léo: Pas officiellement mais on sentait bien cette chose-là. Mon père avait à l'époque une relativement bonne paie. En étant séparé, mais dans la même maison, on voyait bien qu'il $\mathrm{y}$ avait des différences. Il avait plus d'argent que ma mère et puis ma mère assurait surtout l'essentiel. Alors que le père, il pouvait peut-être assurer [...] le superflu. Alors, il avait peut-être le rôle de gentil là-dedans, je dirais. Sans avoir manqué de rien spécialement, [...] on sentait qu'il y avait un souci.

L'observation de ce que leurs parents laissent entrevoir de leur organisation financière et de leur niveau de vie semble centrale dans le processus de socialisation économique. Mais qu'observent donc les enfants? 
La gestion prudente des parents est un élément qui revient régulièrement dans les discours. Dans la plupart de ces familles, le père était le seul pourvoyeur de revenus, la mère ayant généralement cessé son activité professionnelle à l'arrivée des enfants. Ce modèle était largement répandu en Suisse dans les années 1970 et $1980^{6}$. Les mères des classes moyennes qui retournaient sur le marché du travail, le plus souvent à temps partiel $^{7}$, le faisaient généralement lorsque les enfants étaient adolescents. En ce sens, la plupart des personnes interrogées ont grandi dans des familles bénéficiant de l'apport d'un seul revenu, ce qui nécessitait une certaine prudence sur le plan financier. Les propos de Laure synthétisent bien cela:

Mes parents sont très attentifs. Ils ont toujours bien calculé, bien géré, fait attention. Faire attention à leur budget en général, je pense qu'ils devaient aussi le faire. Ma mère a travaillé seulement quand j'avais 15 ans. Donc pour une famille de quatre, ils ont dû bien tout calculer. On m'a transmis ça: la valeur [de l'argent]; que c'est des choses qu'il faut faire attention; qu'on ne dépasse jamais la ligne rouge. Jamais. On n’emprunte pas. Mon père est contre les emprunts. Pas d'emprunt bancaire ou après, il faut rembourser. Ça, c'est exclu.

Comme le souligne Dunand (1998), dans un contexte où il y a peu d'échanges oraux sur le sujet, les enfants doivent repérer les indices leur permettant d'évaluer le niveau de vie de leur famille. Un de ces indices est la manière dont leurs parents réagissaient au désir d'achat qu'ils exprimaient:

Quand on demandait qu'ils nous achètent quelque chose, ils nous disaient: «Non, on n'a pas assez de sous.» Donc, je pense qu'on n'était pas une famille aisée. On n'a manqué de rien mais il fallait faire attention mais sans trop le montrer. Donc, je ne sais pas grandchose. (Nicole)

Les arguments mobilisés par les parents pour accepter ou refuser les demandes d'achats des enfants permettent également à ces derniers d'apprendre à distinguer deux types de dépenses: les dépenses utiles liées à ce que les parents considèrent comme des besoins, et les dépenses secondaires ou superflues qu'ils estiment accessoires au bienêtre. La distinction de ces dépenses implique également des financements différents. Si les parents prennent en charge ce qu'ils considèrent comme relevant des besoins de l'enfant, nous verrons par la suite que l'accès au superflu dépend de la capacité de ce dernier à le financer.

J'ai souhaité des trucs que je n'ai pas reçus comme tout le monde mais je n'étais pas une fille gâtée. Mes parents avaient des moyens mais des moyens modestes, je pense rétrospectivement. Je ne recevais pas des cadeaux comme ça parce que c'est un jour où on

6. «En 1970, les trois quarts environ des couples avec des enfants de moins de sept ans vivaient selon le modèle du père travaillant à plein temps et de la mère au foyer; en 1990, leur proportion était encore de plus de $60 \%[\ldots] »$ (Office fédéral de la statistique, 2005: 50).

7. C'est encore le cas aujourd'hui. Malgré un fort taux d'activité net (76,5\% pour les 15-64 ans), environ $60 \%$ des femmes sont professionnellement actives à temps partiel en 2011. (www.bfs.admin.ch/ bfs/portal/fr/index/themen/03/02/blank/key/erwerbsquote.html; www.bfs.admin.ch/bfs/portal/fr/index/ themen/20/05/blank/key/erwerbstaetigkeit/teilzeitarbeit.html, pages consultées le 9 février 2012). 
reçoit des cadeaux. Je recevais des cadeaux pour mon anniversaire, pour Noël mais pas en dehors. C'est pour ça que je dis modeste. Et puis, je ne recevais pas un pull parce qu'on passait devant un magasin et que le pull plaisait soit à ma mère soit à moi. Il fallait attendre un événement ou il fallait qu'il y en ait besoin. (Alice)

Pour acquérir du sens, l'observation nécessite plusieurs étapes. Les enfants doivent apprendre quoi observer (les indicateurs du niveau de vie familial sont, nous l'avons vu, subtils) et ensuite, ils doivent évaluer ce qu'ils ont observé afin de lui donner une signification. Pour cela, il leur faut comparer leurs observations. Or le monde extérieur vient aux enfants par différentes voies (Hoschschild, 2003). Ainsi, les proches (grandsparents, frères et sœurs, oncles et tantes) et les amis représentent une source importante d'informations. Beaucoup de personnes interrogées relèvent le rôle central des fêtes de famille et notamment de Noël qui, avec son échange de cadeaux, permet d'évaluer le niveau de vie de la famille et de mieux saisir les règles de justice (Kellerhals et al., 1997) qui régissent la distribution des présents.

On avait plein de cadeaux, ce n'était pas des cadeaux qui devaient coûter très cher, ce n'était pas des marques mais c'était l'euphorie, il y avait des papillotes. (Sonia)

Les règles de justice varient selon les familles, Kellerhals et ses collègues (1997) l'ont déjà démontré pour la Suisse. Chez les unes, il s'agit de respecter une stricte égalité entre frères et sœurs. Chez les autres, c'est le principe statutaire, du besoin ou encore du mérite qui règle la distribution des ressources. L'observation de la distribution des biens, notamment à Noël, permet aussi aux enfants d'appréhender les modes de consommation valorisés par les membres de la famille:

Je me souviens même d'avoir reçu des choses qu'elle [ma mère] m'avait déjà offertes ou qui appartenaient à mon frère mais qu'on ne se servait plus et auxquelles on ne faisait plus attention. Pour dire: «Tu vois, tu veux toujours plus mais tu as les choses sous les yeux et tu ne les utilises pas.» (Max)

Si les enfants se comparent à leurs frères et sœurs pour comprendre les règles de justice propres à leur famille, ils se comparent à leurs pairs pour évaluer leur niveau de consommation. Les copains sont des références extrêmement sollicitées durant cette période de la vie. Comme l'ont montré les premières recherches sur le sentiment de justice (dont Homans, 1974), on se compare plus volontiers à ses semblables (pairs ou frères et sœurs) qu’à des personnes de statut nettement supérieur ou inférieur.

Et par rapport aux copains aussi, on avait à peu près la même chose que les autres. On était dans la moyenne. (Marie)

L'observation permet aux enfants d'évaluer le niveau économique de leur famille, de la situer sur l'échelle sociale et de s'informer des règles de justice et des modes de consommation privilégiés. Elle leur offre aussi la vision d'un rapport genré à l'argent. Les enfants de cette époque apprennent que les hommes et les femmes ont des rôles économiques distincts et que l'argent leur fournit un statut différent. 
Dans de nombreuses familles, les mères administrent le quotidien alors que les pères s'occupent des factures. Généralement, elles reçoivent de leur mari une certaine somme pour les dépenses courantes qu'elles gèrent parcimonieusement.

Ils avaient des enveloppes. Ils avaient un budget. Je pense qu'ils mettaient pour chaque chose dans des enveloppes. Après, elles étaient trafiquées pour mettre dans l'autre [rires]. [...]. Hop, [ma mère] prenait dans les enveloppes cadeaux et hop, il n'y a plus [rires]. Et après elle remettait. J'entendais surtout parler de ces enveloppes. (Danièle)

Les stratégies maternelles de gestion du budget, comme la pratique des enveloppes ou la tenue d'une comptabilité domestique, sont perceptibles par les enfants. Un certain nombre d'entre eux a toutefois l'occasion de noter que le statut de gestionnaire de leur mère n'implique pas nécessairement autant de pouvoir et de privilèges économiques que le statut paternel de pourvoyeur des revenus. À l'instar de Jacques, ils sont plusieurs à relever le contrôle exercé par leur père sur l'argent géré par leur mère ou encore son droit à de l'argent de poche (Nyman et Dema, 2007).

Moi, les souvenirs que j'ai, c'est que c'est mon père qui avait droit à de l'argent de poche [...]. Je pense que c'était pour les cafés et les choses comme ça. C'était vraiment pour... les petits plaisirs du mois. (Jacques)

Bien que des tensions familiales - que l'on peut attribuer au décalage entre un contexte politique et social de plus en plus favorable à l'égalité entre les sexes ${ }^{8}$ et des pratiques privées en contradiction — sont relevées, la domination économique vécue par certaines femmes semble peu remise en question.

L'argent, ça a toujours été assez difficile. Pas forcément le fait qu'on n'en ait pas assez mais plutôt que mon père donnait tant à mère et qu'elle avait très peu de liberté à ce niveau-là. [...] Mon père était plus vieux que ma maman et il était entre guillemets encore de l'ancienne époque [...]. C'est vrai que c'était le patriarche, c'était lui qui gérait et ma maman... C'était le début de l'émancipation de la femme et elle a aussi été active làdedans... pour aussi se libérer de cette emprise et puis voilà quoi... c'est vrai que c'était... non pas tabou... mais problématique. (Maurice)

Les rapports de pouvoir ne sont pas discutés au sein de la famille mais ils sont perceptibles. Le retour de leur mère sur le marché du travail est ainsi perçu par les personnes interrogées comme un outil d'émancipation. Le lien entre argent et autonomie féminine apparaît explicitement dans les discours. L'exemple de leur mère a sensibilisé un certain nombre de femmes interrogées à l'importance du revenu féminin dans l'équilibre des rapports de pouvoir conjugaux (Bachmann, 2009; Henchoz, 2008a, 2008b).

Tom: C'est lui qui réglait l'aspect comptable et puis elle, vraiment sa partie, c'était uniquement ce qui concernait le ménager entre guillemets. C'était un peu flou visiblement. Parce qu'acheter des habits, ça fait partie du ménager. Enfin tu vois, il y a des zones à négocier.

8. L'égalité des sexes est inscrite dans la Constitution fédérale en 1981. Le nouveau droit de la famille (1978) et du mariage (1988) garantissent l'autorité parentale conjointe et l'égalité des sexes dans la famille. 
Question: Tu as été témoin de négociations là autour?

Tom: Ouais, bien sûr. Assez régulièrement, mon père trouvait que ma mère était peu économe. En tout cas, ce qu'il remettait en question c'est ce qu'elle achetait, justement en prétextant qu'à son avis, ça ne faisait pas partie [...] du budget ménage.

Question: Elle avait de l'argent personnel, de l'argent de poche?

Tom: Aucune idée alors. Ça non, je ne pourrais pas te répondre... Comme à un certain moment quand on était adolescents, voire même plus tard, elle a commencé à travailler, je suppose que c'était une façon pour elle justement de gagner un peu d'indépendance au niveau financier. J'en déduis que, si elle avait par hasard une somme allouée, elle ne devait pas être énorme.

Dans un tel contexte, les enfants apprennent que l'argent n'a pas toujours la même valeur. Selon sa provenance, l'argent n'a pas la même signification et il ne permet pas d'accéder au même statut. L'argent donné est un argent modeste, fractionné, dont l'utilisation s'opère sous la surveillance du donateur. L'argent gagné par le travail, au contraire, permet d'accéder à l'indépendance et à l'autonomie. Cette distinction conduit Langevin (1996) à différencier l'argent mineur et dépendant (celui des femmes et des enfants), de l'argent adulte et autonome (celui des hommes).

\section{L'expérimentation ou les outils parentaux de la transmission}

Un élément central du processus de socialisation économique est l'apprentissage par l'expérimentation. Des expérimentations encadrées, voire régulées, par les parents qui disposent d'un certain nombre d'outils très concrets pour socialiser l'enfant à l'argent, et ce, dès sa naissance.

\section{Le compte d'épargne jeunesse}

La plupart des parents ont ouvert un compte d'épargne au nom de leur enfant à sa naissance. À l'époque, on parle plutôt de livret d'épargne, car le compte est matérialisé par un livret détenu par l'épargnant où sont notés les dépôts, les retraits et le solde. L'épargne enfantine est également encouragée par les banques suisses qui lui octroient, aujourd'hui encore, un intérêt plus élevé que celui qui est accordé aux comptes pour adultes.

J'avais un compte en banque à la R. C'était mes parents qui mettaient quelque chose dessus et j'ai reçu le solde à mes dix-huit ans. C'était la mode à l'époque. (Max)

Ce carnet d'épargne est accompagné d'un «rapport très physique à l'argent », comme le souligne Cécile. Il est nourri par les versements des parents, voire d'autres proches comme les grands-parents, mais aussi par les enfants eux-mêmes. En effet, ces derniers apprennent très tôt à «mettre de côté» l'argent qu'ils reçoivent pour leur anniversaire ou d'autres fêtes. Le rituel est souvent le même. L'argent est mis dans une tirelire et lorsqu'une certaine somme est atteinte, l'enfant va avec ses parents à la banque la verser sur son livret d'épargne. 
On a eu ces fameuses tirelires en [forme de] dollar. Puis de temps en temps, on allait amener ça à la banque. Je m'en souviens. Mais je n'aimais pas trop mettre là-dedans. (Jules)

Les dépôts et retraits d'argent sont contrôlés par les parents. En effet, toutes les opérations bancaires doivent être effectuées en leur présence, les enfants n'ayant légalement pleinement accès à leur compte qu'à leur majorité (18 ans).

On avait un compte épargne mais qui était géré par mes parents. Et pour l'argent que j'ai reçu à partir du moment où j'ai commencé à travailler [...], je pense qu'on mettait l'argent sur ce compte bancaire et pour le retirer, il fallait que papa soit avec. C'était très géré de cette manière-là parce qu'à l'époque il n'y avait pas possibilité de faire autrement. (Charles)

Grâce à ces pratiques, les enfants apprennent deux choses. Premièrement, ils apprennent à épargner avant même d'apprendre à consommer. Peut-être à cause de la prédominance du protestantisme dans les cantons romands d'où viennent les personnes interrogées, les comportements d'épargne sont inculqués dès le plus jeune âge (Weber, 1999 [1904]). Les sommes que les enfants sont autorisés à dépenser (issues essentiellement de l'argent de poche, nous le verrons) sont en effet généralement bien plus modestes que celles qu'ils épargnent. Deuxièmement, les enfants apprennent à «marquer» l'argent pour reprendre les termes de Zelizer (2005), c'est-à-dire à distinguer des argents différents en leur attribuant des usages différents. Ainsi, l'argentcadeau est distingué de l'argent de poche. Le premier est destiné à l'épargne et le second, nous le verrons, à la consommation. Les sommes que les enfants économisent dès leur plus jeune âge ont pour objectif d'assurer leur avenir et leur entrée dans le monde des adultes. Victor, tout comme Jules, se souvient que son père lui disait: «Il faut mettre de l'argent de côté si, à 18 ans, tu veux t'acheter une voiture.»

J'ai un enregistrement de moi qu'un copain avait fait. J'avais reçu 20 francs pour mes huit ans et puis il m'avait demandé ce que je voulais en faire. J'avais dit: «Je le garde de côté pour m'acheter une ferme plus tard.» (Agathe)

Si certains débitent leur compte épargne avant dix-huit ans, c'est avec l'accord des parents. Et généralement, il s'agit de dépenses présentées comme «utiles», par exemple une trompette ou un vélomoteur. Ce compte n'est jamais débité pour des frais modestes ou de la consommation courante, ceux-ci étant financées par l'argent de poche.

\section{L'argent de poche}

Comme l'a déjà relevé la littérature scientifique (Barnet-Verzat et Wolff, 2001), l'argent de poche versé par les parents apparaît assez tôt dans la vie des enfants (vers 8 ans, voire avant). Il s'agit de petits montants qui permettent de compléter la consommation courante en finançant des dépenses que les parents ne jugent pas essentielles. 
Je n'avais pas tant par semaine mais je me rappelle d'avoir reçu de temps en temps un petit sou pour m'acheter un petit chewing-gum, un petit machin, un petit bois de réglisse au petit kiosque à côté de l'école mais je n'avais pas, ce n'était pas instauré tant. (Annie)

Avec l'âge, les sommes augmentent progressivement. Elles couvrent de plus en plus de frais rattachés à des besoins qui étaient autrefois pris en charge par les parents (BarnetVerzat et Wolff, 2001).

Question: Tu disais que l'argent de poche augmentait petit à petit. Pourquoi est-ce que tes parents l'augmentaient? Est-ce qu'il y a avait une raison derrière?

Marie: Je pense qu'ils se rendaient compte que nous avions plus de besoins. Plus on était grands, plus on voulait de choses.

L'objectif semble donc bien ici d'apprendre aux enfants à devenir des consommateurs autonomes et responsables.

J'ai trouvé assez sympa le fait de nous faire participer à l'argent de poche. De dire: «Chaque mois, on te donne une certaine somme d'argent et c'est à toi de la gérer». [...] C'était une certaine somme et on devait se débrouiller avec ça. Comme ça, on n'allait plus piailler vers nos parents: «Donne-moi un franc et puis un franc.» On avait notre argent et puis voilà. (Marcel)

L'apprentissage ne vise pas seulement à gérer de manière plus autonome sa propre consommation. Il s'agit également de s'émanciper progressivement de la tutelle des parents puisqu'un certain nombre de dépenses n'ont plus besoin de recevoir leur accord:

Avoir mon propre argent, pour moi ça signifiait pouvoir m'acheter ce que, moi, j'avais envie sans que ma maman me dise: «Non, tu ne vas pas pouvoir t'acheter de bonbons.» C’était une petite sorte de liberté. (Marie)

L'argent de poche vise aussi à encourager l'acquisition de compétences en matière de gestion financière. En effet, certains parents conditionnent son versement à la tenue d'une comptabilité simplifiée qu'ils vérifient régulièrement. Les enfants apprennent ainsi à établir un budget et à reporter régulièrement leurs revenus et leurs dépenses.

On recevait de l'argent de poche depuis le début de l'école et jusqu'à la fin des études. Tout au début, on mettait ça dans une grosse tirelire et du moment où on a pu un peu gérer nous-mêmes, j'ai très vite fait des comptes, additionné ce que j'avais, calculé ce qu’il me restait... Je ne les dépensais pas volontiers, j’étais plutôt économe. (Marcel)

Cette exigence parentale, relevée dans les premiers temps du versement de l'argent de poche, est souvent abandonnée par la suite, laissant aux enfants le choix de poursuivre ou non cette pratique. Notons toutefois que le fait que l'argent de poche couvre de plus en plus de dépenses relevant de la consommation courante encourage une gestion prudente. En effet, lorsqu'on est petit, une mauvaise administration de ses ressources a un très faible impact sur son bien-être personnel. Par contre, les compétences de gestionnaire deviennent de plus en plus essentielles à mesure que l'argent de poche couvre davantage de besoins. 
Les parents développent d'autres moyens plus indirects pour inculquer aux enfants une certaine indépendance économique, par exemple en versant des sommes d'argent de poche relativement modestes. Les enfants qui souhaitent augmenter leur consommation sont alors encouragés à chercher d'autres sources de financement.

Si tu veux quelque chose, il faut que tu travailles, il faut le gagner. C'était un petit peu dans leur vision de l'éducation. Si tu veux quelque chose, tu peux travailler. (Yves)

\section{Le «petit job»}

Dès 1877 en Suisse, l'âge minimum du travail des enfants dans les fabriques a été porté à 14 ans. Au xx ${ }^{\mathrm{e}}$ siècle, cette limite sera progressivement fixée à 15 ans (Hauser, 1956). Cependant, à partir de 13 ans, les adolescents sont autorisés par la loi à accomplir des «travaux légers», comme des emplois pendant les vacances. Selon le Département fédéral de l'économie (2010:8), «la distinction entre travail léger et une activité «normale» réside dans la nature et les conditions de l'exercice du travail en question (horaire, fréquence, etc.) ».

Cette législation a contribué à favoriser l'insertion temporaire et progressive des jeunes dans le monde du travail. Excepté deux femmes nées à l'étranger et deux autres qui ont commencé un apprentissage très jeunes, la totalité des personnes interrogées ont travaillé dès l'âge de 13 ans. Il s'agissait d'emplois de vacances effectués dans des usines, des garages, des bureaux ou encore de petits jobs plus réguliers durant l'année comme des gardes d'enfants, des travaux de ferme ou de conciergerie. Certains affirment même avoir travaillé plus jeunes. Dès l'âge de huit ans, Jules recevait une modeste rémunération pour l'aide qu'il apportait dans un garage et Tom a fait ses premières vendanges à douze ans. Dès l'adolescence, la plupart des personnes interrogées gagnent des revenus. Contrairement aux revenus des aînés qui étaient reversés totalement ou partiellement à la mère (Henchoz et Poglia Mileti, 2012), ces gains sont destinés à leur propre consommation. Généralement, ils servent à financer les dépenses qui ne sont pas prises en charge par les parents.

Du fait que mes parents avaient très peu de moyens financiers, je crois qu'ils n'ont pu quasiment rien me financer. Les loisirs et autres, je les ai financés moi-même dès que j'ai pu. Donc, dès que j'avais l'âge, 15-16 ans, j'ai fait un tas de jobs. J'ai fait des études jusqu'à 18 ans-18 ans et demi, mais $j$ 'ai fait des emplois temporaires pendant les vacances qui m'ont permis d'acheter ce que je voulais. (Maurice)

C'est sur ce point que l'on trouve les principales différences de socialisation entre filles et garçons. Les filles ont souvent occupé des petits jobs liés aux relations sociales et à la sphère privée: du baby-sitting ou des emplois dans l'administration. Les garçons mentionnent quant à eux des travaux effectués hors du foyer: travaux de ferme ou de conciergerie (Bihr et Pfefferkorn, 2002). Cette socialisation différenciée au travail rémunéré se confirmera par la suite lorsque ces jeunes devront choisir des métiers (Branger, 2008). Les dépenses financées par ces petits jobs sont également différentes, davantage de femmes mentionnant des frais liés à l'apparence (vêtements, soins du 
corps, etc.). Ce type de consommation peut s'interpréter comme le moyen de marquer son entrée dans le monde adulte, ces dépenses étant pour certaines femmes rattachées à l'accès et à l'expression de la féminité (Henchoz, 2008a).

La principale dépense financée par les petits jobs reste toutefois et ce, autant pour les filles que pour les garçons, l'acquisition d'un moyen de locomotion ou le financement de vacances avec des amis, ce qui permet aux jeunes de prendre de l'autonomie par rapport à leurs parents.

Question: Et cet argent que tu recevais en travaillant, tu avais des idées précises comme pour le vélomoteur, tu savais ce que tu allais en faire?

Mila: Je n'avais pas forcément d'idées précises, mais je me disais: «Ah, si j'ai 500 francs, je peux prévoir de partir en vacances avec des copains ou ce genre de chose. C'était plus la possibilité de pouvoir m'offrir des choses. [...] J'ai ouvert un compte jaune [compte postal] et j'ai mis là-dessus. J'étais assez à essayer d'économiser un max et mettre de côté.

Question: Pourquoi?

Mila: Le fait de savoir que j'avais des sous, puis que je pourrais si je voulais m'acheter quelque chose, c'est plus une idée qui me rassurait. [...] C'était la liberté mais en même temps la possibilité de pouvoir potentiellement m'acheter quelque chose. Mais quand j'achetais quelque chose, ce n'était pas ça qui me satisfaisait le plus. C'était l'idée de me dire: «Si je veux, je peux.»

Selon nous, c'est par le petit job que commence le «processus d'adultification» des rapports à l'argent ${ }^{9}$. L'argent perd de sa connotation enfantine et familiale. Il symbolise désormais l'indépendance économique, la prise en charge de ses propres dépenses et l'accès à l'autonomie. Il incarne la capacité des jeunes à gagner de l'argent et à le gérer. En ce sens, le petit job n'est pas forcément rattaché à un désir de consommation. Il s'agit parfois uniquement de «ressentir plus d'indépendance», comme le rapporte Sybil. Ainsi, il semble surtout symboliser l'entrée des jeunes dans le monde des adultes.

Puis serveuse un mois à Val D’IIliez. Il me semblait que je devenais grande et que c'était normal que j'essaie de trouver un travail pour l'été. [...] Je ne crois pas que j'avais envie de me payer quelque chose. C'était vraiment parce que je sentais que le moment était venu de commencer à m'assumer, dans une certaine mesure. (Cécile)

Aucun de ces adolescents d'alors ne mentionne avoir rendu des comptes sur l'usage de cet argent ou payé une pension par exemple. Le fait que ces revenus soient temporaires l'explique peut-être mais on peut aussi le voir comme une stratégie de la part des parents visant à encourager leur enfant à prendre son indépendance financière et son autonomie. Un certain nombre d'entretiens souligne d'ailleurs le soutien parental à

9. Pour Langevin (1996), c'est la bancarisation de l'argent et l'accès à des revenus stables et réguliers qui marquent la transformation de l'argent juvénile en argent adulte. Le premier point a moins de sens dans le contexte suisse où la bancarisation de l'argent des enfants a lieu dès leur naissance. Par contre, le second point se confirme, d'autant plus qu'en Suisse le faible taux de chômage de l'époque et encore d'aujourd'hui (Sheldon, 2010) conduit à une insertion professionnelle des jeunes plus linéaire et moins fragmentée qu'en France. 
l'entrée des jeunes sur le marché du travail. Par exemple, des pères vont mobiliser leurs relations professionnelles, amicales ou familiales pour trouver un petit job à leur enfant. Comme le relève Agnès, cela «fait partie de l'éducation »:

Très vite, à l'école secondaire, j'ai été en usine; deux — trois fois dans une imprimerie. Faire du travail de secrétariat, du triage de dossiers. Ma tante était infirmière chef dans un hôpital. Elle me prenait avec et puis j'allais deux — trois semaines. Je devais faire des photocopies, des dossiers. Ça ne m'a jamais ennuyée mais je pense que mes parents m'ont un peu poussée à ça. Ils pensaient que c'était bien, que j'avais l'âge.

Les sommes d'argent auxquelles les enfants ont accès ne sont pas traitées de la même manière par les parents. En ce sens, on peut considérer que, par leurs interventions, les parents construisent différents types de monnaies (Zelizer, 2005). Au sein du couple parental, les enfants observent que l'argent reçu peut être légitimement contrôlé par le donateur, nous l'avons vu. Ce constat est confirmé par la manière dont les parents traitent l'argent que les enfants reçoivent en cadeau ou en argent de poche. Ces sommes sont contrôlées par les parents qui en règlent l'accès aux enfants. Dans les entretiens, le rôle des pères et des mères n'est pas toujours précisé. Toutefois, le versement d'argent de poche et le suivi des comptes semblent davantage la responsabilité des mères, notamment parce que cela est perçu comme relevant de leur sphère de compétences (éducation et tenue du budget courant). Les pères de leur côté semblent jouer un rôle plus actif dans l'intégration des enfants sur le marché du travail, ce qui peut s'expliquer par le fait qu'ils sont bien plus nombreux à être insérés professionnellement.

Au contraire de l'argent reçu, l'argent gagné par le labeur est laissé libre d'usage, les parents intervenant très peu dans ce domaine. Cette absence d'intervention peut être considérée comme une manière d'encourager les adolescents à entamer un processus d'émancipation. D'une part, en gagnant de l'argent qu'ils sont libres de gérer selon leurs désirs et d'autre part, en utilisant cet argent pour acquérir les outils de leur autonomie, comme des moyens de locomotion ou des vacances entre amis. En n'intervenant pas sur l'argent gagné par le travail et en les encourageant à entrer dans le monde professionnel, les parents contribuent également à socialiser leurs enfants au travail rémunéré et à leur signifier sa valeur centrale dans l'accès à l'autonomie et plus largement dans l'accès à un statut social et à une identité adultes (Lalive d'Épinay et Garcia, 1988).

\section{CONCLUSION}

Comme l'a relevé la littérature scientifique, les parents jouent un rôle déterminant dans la socialisation économique. Leurs enfants retiennent toutefois moins leur rôle d'éducateurs que les messages plus ou moins diffus qui leur sont adressés autour de l'épargne, la production de revenu et la consommation. En effet, s'ils éduquent par l'argent de poche, les parents ont un rôle plus large dans le processus de socialisation économique. Un rôle qui leur échappe en partie, car les enfants ne se contentent pas 
de ce qu'on leur enseigne. Ils piochent aussi un certain nombre d'informations dans leur entourage. L'entourage ne fait pas que fournir des informations, il permet aussi de les interpréter par la comparaison. Ainsi, en observant leurs parents, les enfants apprennent que l'argent n'est pas une affaire dont on débat sur la scène publique. C'est une affaire qui relève plus spécifiquement de la sphère conjugale, voire individuelle. Les enfants apprennent la discrétion et les mesures subtiles du niveau de vie qui nécessitent de comparer régulièrement les pratiques et consommations familiales à celles des voisins ou des pairs. Les enfants apprennent que l'argent est une chose sérieuse qu'on gère sérieusement. Si les pères sont les pourvoyeurs de revenus, les mères jouent un rôle central dans l'équilibre budgétaire en gérant parcimonieusement le budget quotidien. Les parents sont également les accompagnateurs des expérimentations financières enfantines. Cet accompagnement encourage la parcimonie, le travail et l'épargne. Dans la continuité de la répartition sexuée du travail entre les conjoints, ce sont souvent les mères qui apprennent aux enfants à gérer les revenus et les pères qui mobilisent leurs relations professionnelles pour encourager l'entrée sur le marché du travail. La manière dont les parents interviennent sur les différents types d'argent en possession des enfants contribue à construire des catégories spécifiques de monnaies (Zelizer, 2005) : l'argent donné s'accompagne d'un contrôle parental plus ou moins explicite. Les enfants apprennent ainsi que la gratuité ne va pas de soi, que l'argent est un instrument de pouvoir et un outil de contrôle social (Hertz, 1992). L'absence de contrôle parental sur l'argent gagné par le travail revient d'une part, à associer l'argent gagné par l'effort à l'indépendance et à l'autonomie et d'autre part, à affirmer la vertu du travail rémunéré. En entendant ces personnes qui ont grandi dans les années 19701980, on ne peut être que surpris de la force de ce message que Max Weber (1999 [1904]) a associé à l'éthique protestante calviniste et que plus récemment Boltanski (1966: 38) a attribué aux valeurs traditionnelles suisses. Selon lui, les «vertus suisses» comme le travail, l'ordre et l'économie «tiennent toutes entières dans l'opposition entre le sérieux et le futile, dichotomie qui vaut pour toutes les situations de l'existence [...]. Être travailleur, c'est d'abord manifester du sérieux dans son travail à la façon dont être propre ou économe, c'est manifester du sérieux dans la tenue de son ménage» (Boltanski, 1966: 24).

Est-ce cela qui explique que les Suisses sont aujourd'hui parmi les moins endettés (Fleury et Christin, 2011) et les plus épargnants d'Europe ${ }^{10}$ ? Cet article ne permet malheureusement pas d'intégrer dans l'analyse ce que ces enfants, aujourd'hui adultes, ont fait de cet héritage. On peut toutefois en relever des traces. La persistance à l'âge adulte de la dichotomie entre le sérieux/nécessaire et le futile/superflu comme critères d'achat en est une. Le rattachement symbolique de l'argent gagné à l'autonomie et à l'indépendance et de l'argent donné au contrôle et au devoir de réciprocité, souvent

10. En 2009, le taux d'épargne brut des ménages est de $17.12 \%$ (13,44 en moyenne pour l'U27). C'est un des plus hauts d'Europe (www.epp.eurostat.ec.europa.eu/tgm/table.do? tab=table\&init=1\&langu age $=$ fr\&pcode $=$ tsdec240\&plugin $=1$, page consultée le 3 avril 2012). 
mobilisé comme critères de justification des rapports de pouvoir au sein du couple (Henchoz, 2008a), en est une autre.

\section{RÉSUMÉ}

La socialisation économique est un sujet peu étudié par la sociologie économique, celle de la jeunesse et de la socialisation. Nous l'abordons ici à partir d'un point de vue original, celui des «socialisés » eux-mêmes, ce qui permet de mettre en évidence la part active qu'ils prennent à ce processus. L'analyse de plus de 40 entretiens portant sur l'enfance et l'adolescence d'hommes et de femmes de Suisse francophone aujourd'hui âgés entre 25 et 40 ans met en lumière trois composantes essentielles de l'apprentissage du rapport à l'argent: l'éducation parentale, l'observation et l'expérimentation. Nous montrons que les jeunes apprennent très tôt à devenir des consommateurs et des gestionnaires prudents mais aussi des producteurs de revenus. L'accent porté sur des valeurs comme l'épargne et le travail contribue peut-être à expliquer pourquoi la Suisse est un pays dont le taux d'endettement des ménages est parmi les plus faibles d'Europe.

Mots clés: argent, jeune, socialisation, transmission, famille

\section{ABSTRACT}

The sociology of economics, of youth and of socialization have paid very little attention as yet to economic socialization. Taking into account the point of view of the 'socialized' themselves enables highlighting their active role in the process. The analysis of over 40 childhood and adolescence interviews of men and women from French-speaking Switzerland, between 25 and 40 , shows three essential components of economic learning: parental education, selective observation and experimentation. Young people learn early on how to become careful consumers, managers and producers of money. The emphasis on values such as savings and work contributes to explain why Switzerland is a country where the household debt ratio is among the lowest in Europe.

Key words: money, young, economic socialization, financial education, transmission, family

\section{RESUMEN}

La socialización económica es un tema poco estudiado por la sociología económica, la de la juventud y de la socialización. Aquí la abordamos a partir de un punto de vista original, el de los "socializados", lo que permite poner en evidencia la parte activa que éstos toman en este proceso. El análisis de más de 40 entrevistas acerca de la infancia y la adolescencia de hombres y mujeres en Suiza francófona, cuyas edades oscilan actualmente entre los 25 y los 40 años, revela tres componentes esenciales del aprendizaje de la relación con el dinero: la educación parental, la observación y la experimentación. Aquí mostramos que los jóvenes aprenden muy temprano a devenir consumidores y administradores prudentes, e igualmente productores de ingresos. El énfasis dado en valores tales como el ahorro y el trabajo contribuye quizás a explicar por qué Suiza es un país donde la tasa de endeudamiento de los hogares se encuentra entre las más bajas de Europa.

Palabras claves: dinero, joven, socialización, transmisión, familia 


\section{BIBLIOGRAPHIE}

Bamert, T. et P. Oggenfuss (2005), Ausgabeverhalten von Jugendlichen: Ergebnisse einer Befragung von Jugendlichen im Kanton Zürich: Kurzbericht zur Studie, Zürich, Universität Zürich.

Barnet-Verzat, C. and F.-C. WolfF (2008), «Pocket Money and Child Effort at School», Economics Bulletin, vol. 9, n 13, p. 1-10.

__ (2001), «L'argent de poche versé aux jeunes: l'apprentissage de l'autonomie financière», Économie et statistique, vol. $343, \mathrm{n}^{\circ} 3$, p. 51-72.

Bertaux, D. (1997), Les récits de vie: Perspective ethnosociologique, Nathan, Paris.

Berti, A. E. and A. S. Вомві (1988), The Child's Construction of Economics, Cambridge, Cambridge University Press.

Beutler, I. and L. Dickson (2008), «Consumer Economic Socialization», in J. Xiao (dir.), Handbook of Consumer Finance Research, New York, Springer, p. 83-102.

Binr, A. et R. Pfefferkorn (2002), Hommes-femmes, quelle égalité?, Paris, L'Atelier.

Boltanski, L. (1966), Le bonheur suisse, Paris, Minuit.

Branger, K. (2008), Vers l'égalité entre femmes et hommes. Situation et évolution, Neuchâtel, Office fédéral de la statistique.

Bullock, K. M. et al. (1993), «Economic and Industrial Understanding in the Primary School: An overview ", Early Child Development and Care, vol. 94, n 1, p. 11-20.

Darmon, M. (2006), La socialisation, Paris, Armand Colin.

Dell'Orto, G. and K. O. Doyle (2001), «Poveri Ma Belli, Meanings of Money in Italy and in Switzerland», American Behavioral Scientist, vol. 45, n 2, p. 257-271.

Département fédéral de l'économie — Secrétariat d’état À l'économie (2010), Protection des jeunes travailleurs - Informations pour les jeunes de moins de 18 ans, Berne, SECO, Conditions de travail.

Deutsch, N. L. and E. Theodorou (2010), «Aspiring, Consuming, Becoming: Youth Identity in a Culture of Consumption", Youth and Society, vol. 42, n 2, p. 229-254.

Dubar, C. (1991), La socialisation. Construction des identités sociales et professionnelles, Paris, Armand Colin.

Durkheim, E. (1968 [1922]), Éducation et sociologie, Paris, PUF.

Fleury, S. et T. Christin (2011), Revenus, consommation et fortune - Analyses. Endettement, Office fédéral de la statistique, Neuchâtel, www.bfs.admin.ch/bfs/portal/fr/index/themen/20/02/blank/dos/02.html, page consultée le 14 novembre 2012.

Furnham, A. (1999), «Economic Socialization: A Study of Adults' Perceptions and Uses of Allowances (Pocket Money) to Educate Children ", British Journal of Developmental Psychology, vol. 17, $\mathrm{n}^{\circ} 4$, p. 585-604.

Furnham, A. (2001), «Parental Attitudes to Pocket Money/Allowances for Children », Journal of Economic Psychology, vol. 22, n 3, p. 397-422.

Galland, O. (2009 [1984]), Les jeunes, Paris, La Découverte.

Glaser, B. and A. Strauss (1967), The Discovery of Grounded Theory: Strategies for Qualitative Research, Chicago, Aldine.

Goffman, E. (1959, 1996), La Mise en scène de la vie quotidienne. La présentation de soi, Paris, Minuit.

Hauser, A. (1956), Zur Geschichte der Kinderarbeit in der Schweiz, Zürich, Polygraphischer Verlag.

Henchoz, C. et F. Poglia Mileti (2012), «"Les larmes de ma mère”. Comprendre le processus de démocratisation de l'économie familiale par les contributions financières des femmes et leur perception par les hommes", Revue suisse de sociologie, vol. 38, n 3, p. 401-419.

Henchoz, C. et B. Wernli (2012), «L'endettement des jeunes est-il supérieur à celui des adultes en Suisse?», La Vie économique, revue de politique économique, vol. 1/2, p. 53-56. 
Henchoz, C. (2008a), Le couple, l'amour et l'argent. La construction conjugale des dimensions économiques de la relation amoureuse, Paris, L'Harmattan.

—_, (2008b), «Le rôle de l'indépendance financière dans la construction du lien conjugal contemporain. L'analyse du processus d'individualisation des finances dans trois générations de couples », Revue internationale Enfances, Familles, Générations, vol. 9, p. 36-51.

Hertz, R. (1992), «Financial Affairs: Money and Authority, in S. Lewis et al. (dir.), Dual-Earner Marriage, London, Sage, p. 127-150.

Homans, G. (1974), Social Behavior: Its Elementary Forms, New York, Harcourt Brace Jovanovich Inc.

Hoschschild, A. R. (2003), "Children as eavesdroppers», in A. R. Hochschild (dir.), The Commercialization of Intimate Life, Berkeley, University of California Press, p. 172-181.

Kellerhals, J. et al.,(1997), Le sentiment de justice dans les relations sociales, Paris, Presses universitaires de France.

Lahire B. (2002), Portraits sociologiques. Dispositions et variations individuelles, Paris, Nathan.

Lalive d'Épinay, C. et C. GARCia (1988), Le Mythe du travail en Suisse: splendeur et déclin au cours du XX siècle, Genève, Georg.

LANGevin, A. (1996), «Images symboliques de l'argent et classes d'âge», Agora, vol. 3, p. 11-22.

LEA, S. et al. (1995), "Psychological Factors in Consumer Debt: Money Management, Economic Socialization, and Credit Use», Journal of Economic Psychology, vol. 16, n 4, p. 681- 701.

Leiser, D. (1984), «Children's Conceptions of Economics. The Constitution of a Cognitive Domain», Journal of Economic Psychology, vol. 4, n 4, p. 297-317.

Lemieux, D. et L. Mercier (1989), Les femmes au tournant du siècle, 1880-1940. Âges de la vie, maternité et quotidien, Québec, Institut québécois de recherche sur la culture.

Nyman, C. and S. Dema (2007), "An Overview: Research on Couples and Money», in D. Stocks et al. (dir.), Modern Couples Sharing Money, Sharing Life, New York, Palgrave Macmillan, p. 7-29.

O'Brien, M. (1995), «Allocation of Resources in Households: Children's Perspectives», The Sociological Review, vol. 43, n 3, p. 501-517.

OfFice fÉdÉRAl DE LA STATISTIQUe (2005), Enquête sur les revenus et la consommation 2003, Neuchâtel, Office fédéral de la statistique.

Piaget, J. (1947, 1967), La psychologie de l'intelligence, Paris, Colin.

Рцомв, F. (2005), Faire entrer le travail dans sa vie: vers de nouvelles modalités d'intégration professionnelle des jeunes, Paris, L'Harmattan.

Praz, A.-F. (2005), De l'enfant utile à l'enfant précieux. Filles et garçons dans les cantons de Vaud et Fribourg, 1860 et 1930, Lausanne, Antipodes.

Roland-Levy, C. (2004), «De la psychologie sociale à la psychologie économique en passant par la psychologie politique», Les cahiers psychologie politique, vol. 5, www.lodel.irevues.inist.fr/ cahierspsychologiepolitique/index.php? id=1302, page consultée le 4 novembre 2012.

Sheldon, G. (2010), «Le marché suisse du travail depuis 1920: les tendances à long terme», La Vie économique, vol. 1/2, p. 15-19.

Singly, F. (de) (2000), «Penser autrement la jeunesse», Lien social et Politiques, vol. 43, p. 9-21.

Sonuga-Barke, J. S. and P. Webley (1993), Children's Savings. A Study in the Development of Economic Behavior, Hillsdale, Lawrence Erlbaum Associates.

Streuli, E. et al. (2008), Eigenes Geld und fremdes Geld — Jugendliche zwischen finanzieller Abhängigkeit und Mündigkeit, Basel, Gesowip.

Walser, S. et M. Killias (2009), Jugenddelinquenz im Kanton St. Gallen. Bericht, St. Gallen, Justizdepartements.

Ward, S. (1974), «Consumer Socialization», Journal of Consumer Research, vol. 1, n 2, p. 1-14.

Weber, M. (1999 [1904]), L'Éthique protestante et l'esprit du capitalisme, Paris, Flammarion.

Zelizer, V. (1994), Pricing the Priceless Child: The Changing Social Value of Children. Princeton, Princeton University Press.

Zelizer, V. (2005), La Signification sociale de l'argent, Paris, Seuil. 